\title{
Identifying drug substances of screening tool for older persons' appropriate prescriptions for Japanese
}

\author{
Kaori Nomura', Taro Kojima², Shinya Ishii ${ }^{2}$, Takuto Yonekawa ${ }^{3}$, Masahiro Akishita ${ }^{2}$ and Manabu Akazawa ${ }^{3^{*}}$ (D)
}

\begin{abstract}
Background: In 2015, the Japan Geriatric Society (JGS) updated "the Guidelines for Medical Treatment and its Safety in the elderly," accompanied with the Screening Tool for Older Persons' Appropriate Prescriptions for Japanese (STOPP-J): "drugs to be prescribed with special caution" and "drugs to consider starting." The JGS proposed the STOPP-J to contribute to improving prescribing quality; however, each decision should be carefully based on medical knowledge. The STOPP-J shows examples of commonly prescribed drug substances, but not all relevant drugs. This research aimed to identify substances using such coding, as a standardized classification system would support medication monitoring and pharmacoepidemiologic research using such health-related information.

Methods: A voluntary team of three physicians and two pharmacists identified possible approved medicines based on the STOPP-J, and matched certain drug substances to the Anatomical Therapeutic Chemical Classification (ATC) and the Japanese price list as of 2017 February. Injectables and externally used drugs were excluded, except for self-injecting insulin, since the STOPP-J guidelines are intended to cover medicines used chronically for more than one month. Some vaccines are not available in the Japanese price list since they not reimbursed through the national health insurance.

Results: The ATC 5th level was not available for 39 of the 235 identified substances, resulting in their classification at the ATC 4th level. Furthermore, among 26 combinations, 10 products were matched directly to the ATC 5th level of the exact substances, and others were linked to the ATC representing the combination or divided into multiple substances for classification if the combination was not listed in the ATC.

Conclusion: This initial work demonstrates the challenge of matching ATC codes and the Japan standard commodity classification codes corresponding to STOPP-J substances. Since coding facilitates database analysis, the proposed drug list could be applied to research using large databases to examine prescribing patterns in patients older than 75 years or who are frail. Since ATC is not available for some substances, Japanese medicines need the process to be registered in the ATC for an effective screening tool to be developed for STOPP-J.
\end{abstract}

Keywords: ATC, Geriatric patients, STOPP-J, Database, Appropriate prescribing

\section{Background}

Medication prescribing for the elderly is a complex task that requires special care and increased patient monitoring, while appropriate medications are vital for keeping elderly patients healthy, especially those with multiple diseases who use polypharmacy. Japan is known as the most aged country with $26.5 \%$ of the population older

\footnotetext{
* Correspondence: makazawa@my-pharm.ac.jp

${ }^{3}$ Public Health and Epidemiology, Meiji Pharmaceutical University, Tokyo, Japan

Full list of author information is available at the end of the article
}

than 65 [1]. Therefore, a regulatory meeting was established in 2017 to discuss appropriate prescribing and medication use in the elderly and ensure adaptability to the changing medical status of patients [2]. Furthermore, this strategy is expected to reduce the side effects and polypharmacy and ensure reasonable medical costs [2]. Japan has been releasing new drugs to the world, and its regulatory authorities and pharmaceutical companies are expected to provide enough information and formulate precautions for the use of drugs in the elderly. However, since clinical trials mostly exclude the elderly, clinical

(c) The Author(s). 2018 Open Access This article is distributed under the terms of the Creative Commons Attribution 4.0 International License (http://creativecommons.org/licenses/by/4.0/), which permits unrestricted use, distribution, and reproduction in any medium, provided you give appropriate credit to the original author(s) and the source, provide a link to the Creative Commons license, and indicate if changes were made. The Creative Commons Public Domain Dedication waiver (http://creativecommons.org/publicdomain/zero/1.0/) applies to the data made available in this article, unless otherwise stated. 
information for this demographic is scarce for most new drugs, studies are eagerly anticipated, which is true in every country and region.

To aid physicians to prescribe appropriately, the Japan Geriatric Society (JGS) first published guidelines for safe pharmacotherapy in the elderly, and a list of potentially inappropriate medication uses in 2005 [3], which is recognized as the Japanese version of the Beers criteria [4]. It was updated in 2015, providing the Screening Tool for Older Persons' Appropriate Prescriptions for the Japanese (STOPP-J) for drugs to be prescribed with special caution and drugs to be considered for treatment [5]. Ahead of this, in Europe, the Screening Tool of Older Persons' potentially inappropriate Prescription (STOPP) $[6,7]$, proposed by the Ireland study group, is reported as a useful guide for identifying potentially inappropriate medications, particularly for hospital inpatients [8].

Recent evolutions in medical informatics and computerization have enabled researchers to use various databases and analytical tools in their studies. Studies using the databases of regulatory authorities, insurance claims, and medical records, as well as patients' reports have become popular in public health disciplines and drug development phases recently as well as post-marketing phase. However, there are still challenges associated with the methods of collection, coding, and analysis of data for assessing the accuracy of medication use. The use of identical names or a systematic code for drugs enhances the efficiency of research with large-scale databases [9]. However, coding of medication data depends on the regulatory system, which varies between countries. For example, the National Drug Code in the US (https://www.fda.gov/ Drugs/InformationOnDrugs/ucm142438.htm.) differs from the BNF codes (https://data.gov.uk/dataset/176ae2 64-2484-4afe-a297-d51798eb8228/resource/bac33489-b3dc-47ec-b688-da9cf40e25bd) in the UK. For research on medicine use in the elderly, the AGS Beers criteria [4], STOPP/START [7], and JGS guidelines [5] have been used. However, these guidelines mainly provide drug categories and medication considerations, which lack consistency (Table 1). In addition, they do not specifically define molecular entities, so that researchers have to select drug substances to be studied. Therefore, variation often occurs when using databases to investigate drug use. To facilitate computerized work with databases, Groot et al. [8] proposed a uniform coding for drugs approved in the Netherlands, compliant with the STOPP/START. On the other hand, Japan has several drug-coding systems, depending on the regulatory objective, such as those for labeling information, reimbursement of medical fees, and a third for logistics, but they are solely for domestic use. A variety of proprietary databases is currently available in Japan, and medical terms are mostly compared between them using the International Classification of Diseases (ICD) [10]

Table 1 Characteristics of pharmacotherapy criteria for older adults

\begin{tabular}{|c|c|c|c|}
\hline & AGS Beer's criteria $2015^{1)}$ & STOPP/START V2 $2015^{2)}$ & JGS STOPP-J $2015^{3)}$ \\
\hline $\begin{array}{l}\text { Latest version } \\
\text { Developed }\end{array}$ & AGS & Study group at University College Cork & JGS \\
\hline $\begin{array}{l}\text { Original version } \\
\text { (year, developer) }\end{array}$ & 1991, Dr. Beers & $\begin{array}{l}\text { 2008, Study group at } \\
\text { University College Cork }\end{array}$ & 2005, JGS \\
\hline $\begin{array}{l}\text { Target } \\
\text { population }\end{array}$ & $\begin{array}{l}\text { United States; } 65 \text { and older; Ambulatory, } \\
\text { acute, and institutionalized settings }\end{array}$ & $\begin{array}{l}\text { Europe-wide prescribing practices; } \\
65 \text { and older; in most clinical settings }\end{array}$ & $\begin{array}{l}\text { Japan; } 75 \text { and older; } 74 \text { and } \\
\text { younger with frailty; } 65 \text { and } \\
\text { older in need of nursing care; } \\
\text { chronic treatment }\end{array}$ \\
\hline $\begin{array}{l}\text { Therapeutic } \\
\text { category / drug }\end{array}$ & $\begin{array}{l}26 \text { potentially inappropriate medication (PIM) } \\
12 \text { PIM due to drug-disease/ drug-syndrome interactions } \\
5 \text { PIM to be used with caution } \\
10 \text { PIM non-anti-infective drug-drug interactions that } \\
\text { should be avoided in older adults }\end{array}$ & $\begin{array}{l}80 \text { criteria in STOPP } \\
34 \text { criteria in START }\end{array}$ & $\begin{array}{l}29 \text { groups for drugs to be } \\
\text { prescribed with special caution } \\
8 \text { groups for drugs to } \\
\text { consider starting }\end{array}$ \\
\hline Remarks & $\begin{array}{l}\text { Supplement information: "Quality of evidence" and } \\
\text { "Strength of Recommendation" }\end{array}$ & $\begin{array}{l}\text { For adverse drug events prevention } \\
\text { and cost reduction }\end{array}$ & $\begin{array}{l}\text { Supplement information: } \\
\text { "Quality of evidence" and } \\
\text { "Strength of Recommendation" }\end{array}$ \\
\hline
\end{tabular}

\section{Abbreviations:}

AGS: American Geriatric Society

JGS: Japan Geriatric Society

PIM: Potentially Inappropriate Medication use in older adults

STOPP: Screening Tool of Older People's potentially inappropriate Prescriptions

START: Screening Tool to Alert doctors to Right Treatment

STOPP-J: Screening Tool for Older Persons' Appropriate Prescriptions for Japanese

${ }^{1)}$ American Geriatrics Society. 2015 Beers Criteria Update Expert Panel. American Geriatrics Society 2015 Updated Beers Criteria for Potentially Inappropriate Medication Use in Older Adults. J Am Geriatr Soc. 2015;63:2227-2246

${ }^{2)}$ O'Mahony D, O'Sullivan D, Byrne S, O'Connor M, Ryan C, Gallagher P. STOPP/START criteria for potentially inappropriate prescribing in older people: version 2. Age Ageing. 2015;44:213-218

${ }^{3)}$ Kojima T, Mizukami K, Tomita N, Arai H, Ohrui T, Eto M, et al. Screening Tool for Older Persons' Appropriate Prescriptions for Japanese: Report of the Japan Geriatrics Society Working Group on“Guidelines for medical treatment and its safety in the elderly." Geriatrics Gerontology Int. 2016;16:983-1001 
or the Medical Dictionary for Regulatory Activities (MedDRA) [11], which are internationally recognized in pharmacoepidemiological studies. Unfortunately, due to nationally defined drug coding based on approval, the indications differ, and the classification of medication data may not be consistent across databases. Furthermore, additional efforts for drug identification and matching are necessary when coding systems are different. This process could introduce mismatching and misinterpretation flaws into studies using multiple databases. Therefore, it would be worthwhile to standardize drug codes for international use, e.g., global pharmacovigilance, as the aforementioned Dutch group did by identifying STOPP/START drugs at the substance level and using international coding systems [8]. However, while a local version of the Beers criteria and STOPP/START has been proposed in Japan $[12,13]$, no guidelines have been presented on how to encode medications. In this paper, we present a proposal to encode drugs in JGS medication guidelines using the Anatomical Therapeutic Chemical Classification System (ATC) [14], supporting the extraction and validity of the medication use data.

\section{Methods}

\section{Process for listing drug substances}

This study was set up voluntarily by three physicians (Akishita, Kojima, and Ishii) and two pharmacists (Akazawa and Nomura) who have experience with studies on drug use in the elderly, using both clinical observations and databases. The STOPP-J 2015 developed by the JGS through systematic review, repeated group discussion, and review by the related academic societies, followed by public consultation [15], was the basis for this study. This JGS tool for medications for older persons does not include details on drug dosage, frequency, or duration of administration, but rather includes drug categories or names. The first step involved drafting a list of approved proprietary names based on JGS guidelines with the support of the Japan Pharmaceutical Information Center (JAPIC), an organization that provides drug information and codes to the Ministry of Health, Labour and Welfare, and pharmaceutical companies. JAPIC provided the English and Japanese names of medicinal substances, supervised by Akazawa and Nomura. The National Health Insurance Drug Price List as of February 2017 [16] was referenced for substance names in Japanese. They included all relevant active substances approved in Japan, which were grouped into specific categories, e.g., the statin category included atorvastatin, simvastatin, pitavastatin, pravastatin, fluvastatin, and rosuvastatin.

In the second step, the physicians, Kojima and Ishii, reviewed the drafted drug names in parallel from the perspectives of clinical treatment of the elderly, supervised by Akishita to reach a consensus. Since the JGS guidelines suggest controlling long-term medication for older persons to avoid untoward systemic adverse events occurring in any case where a drug is used or unused, external drugs and injections were excluded, except for self-injection. Simultaneously, the pharmacists matched the Japanese drug names with the national price list and the English drug names with ATC codes according to pharmacological criteria. Yonekawa helped encoding work. All possible oral indications were considered. Table 2 shows the criteria used to choose substances, categories, and codes.

\section{Drug classification systems}

There are two major global drug classification systems; the Anatomical Therapeutic Classification by the European Pharmaceutical Market Research Association (EPhMRA) [17] and the ATC created by the World Health Organization (WHO) Collaborating Centre for Drug Statistics [14]. Our study used the WHO ATC classification, which has codes at the substance level known as the 5 th level.

In Japan, several drug-coding systems exist. The Japan standard commodity classification includes classes for drugs, which appear similar to the 3rd level of the ATC. However, it should be used with caution since it has not

Table 2 Procedures and concepts for listing drugs and codes

\begin{tabular}{ll}
\hline Listing drugs & - Listed JGS substances were limited to those approved as medicinal product for oral use in Japan, except insulin. \\
& - Listed JGS substances were prescribed for long-term use in general. \\
& - If a combination drug is comprised with more than one of listed therapeutic category, combinations were presented in Table 5. \\
\hline Coding with & - If ATC codes at the 5 th level cannot be directly matched to the substance, an alternate 4th level code is proposed \\
ATC & by searching online [1]. \\
& - The 5 th level ATC code is proposed when it is available for the combination, otherwise ATC codes are searched for each substance. \\
Coding with & - Some vaccines are not available because vaccine is not covered by the pricing list for the national health insurance but \\
Japan code & by other public support. \\
& - The pricing list is available for medicinal products currently in the market.
\end{tabular}


Table 3 Proposal for drug coding of "List of drugs to be prescribed with special caution" 1)

\begin{tabular}{|c|c|c|}
\hline Therapeutic category/JAN English name & Japan 2) & $\mathrm{ATC}^{3)}$ \\
\hline \multicolumn{3}{|c|}{ Nervous system: Overall antipsychotic drugs - Antipsychotic drugs } \\
\hline Aripiprazole Hydrate & 1179045 & N05AX12 \\
\hline Asenapine Maleate & 1179056 & N05AH05 \\
\hline Blonanserin & 1179048 & N05AX \\
\hline Bromperidol & 1179028 & N05 AD06 \\
\hline Chlorpromazine Hydrochloride & 1171001 & N05AA01 \\
\hline Chlorpromazine Phenolphthalinate & 1171005 & N05AA01 \\
\hline Clocapramine Hydrochloride Hydrate & 1179030 & N05AX \\
\hline Clozapine & 1179049 & N05AH02 \\
\hline Fluphenazine Maleate & 1172009 & N05AB02 \\
\hline Haloperidol & 1179020 & N05 AD01 \\
\hline Levomepromazine Maleate & 1172014 & N05AA02 \\
\hline Mosapramine Hydrochloride & 1179035 & N05AX10 \\
\hline Nemonapride & 1179036 & N05AL \\
\hline Olanzapine & 1179044 & N05AH03 \\
\hline Oxypertine & 1179011 & N05AE01 \\
\hline Paliperidone & 1179053 & N05AX13 \\
\hline Perphenazine & $\begin{array}{l}1172006 \\
1172007\end{array}$ & N05AB03 \\
\hline Perphenazine Fendizoate & 1172004 & N05AB03 \\
\hline Perphenazine Maleate & 1172013 & N05AB03 \\
\hline Perospirone Hydrochloride Hydrate & 1179043 & N05AX \\
\hline Pimozide & 1179022 & N05AG02 \\
\hline Pipamperone Hydrochloride & 1179006 & N05 AD05 \\
\hline Prochlorperazine Maleate & 1172010 & N05AB04 \\
\hline Propericiazine (Periciazine) & 1172005 & N05 AC01 \\
\hline Quetiapine Fumarate & 1179042 & N05AH04 \\
\hline Risperidone & 1179038 & N05AX08 \\
\hline Spiperone & 1179015 & N05 AD \\
\hline Sulpiride & $\begin{array}{l}1179016 \\
2329009\end{array}$ & N05AL01 \\
\hline Sultopride Hydrochloride & 1179032 & N05AL02 \\
\hline Tiapride Hydrochloride & 1190004 & N05AL03 \\
\hline Timiperone & 1179026 & N05 AD \\
\hline Zotepine & 1179024 & N05AX11 \\
\hline \multicolumn{3}{|l|}{ Combination (see Table 5) } \\
\hline \multicolumn{3}{|l|}{ Nervous system: Benzodiazepines } \\
\hline Alprazolam & 1124023 & N05BA12 \\
\hline Bromazepam & 1124020 & N05BA08 \\
\hline Brotizolam & 1124009 & N05CD09 \\
\hline Chlordiazepoxide & 1124028 & N05BA02 \\
\hline Clorazepate Dipotassium & 1124015 & N05BA05 \\
\hline Clotiazepam & 1179012 & N05BA21 \\
\hline Cloxazolam & 1124014 & N05BA22 \\
\hline Diazepam & 1124017 & N05BA01 \\
\hline
\end{tabular}


Table 3 Proposal for drug coding of "List of drugs to be prescribed with special caution" ${ }^{1)}$ (Continued)

\begin{tabular}{|c|c|c|}
\hline Therapeutic category/JAN English name & Japan ${ }^{2)}$ & ATC $^{3)}$ \\
\hline Estazolam & 1124001 & N05CD04 \\
\hline Ethyl Loflazepate & 1124029 & N05BA18 \\
\hline Etizolam & 1179025 & N05BA19 \\
\hline Fludiazepam & 1124019 & N05BA17 \\
\hline Flunitrazepam & 1124008 & N05CD03 \\
\hline Flurazepam Hydrochloride & 1124002 & N05CD01 \\
\hline Flutazolam & 1124024 & N05BA \\
\hline Flutoprazepam & 1124027 & N05BA \\
\hline Haloxazolam & 1124005 & N05CD \\
\hline Lorazepam & 1124022 & N05BA06 \\
\hline Lormetazepam & 1124010 & N05CD06 \\
\hline Medazepam & 1124021 & N05BA03 \\
\hline Mexazolam & 1124025 & N05BA \\
\hline Nimetazepam & 1124004 & N05BA \\
\hline Nitrazepam & 1124003 & N05CD02 \\
\hline Oxazolam & 1124013 & N05BA \\
\hline Quazepam & 1124030 & N05CD10 \\
\hline Rilmazafone Hydrochloride Hydrate & 1129006 & N05CD \\
\hline Tofisopam & 1124026 & N05BA23 \\
\hline Triazolam & 1124007 & N05CD05 \\
\hline \multicolumn{3}{|l|}{ Nervous system: Non-benzodiazepines } \\
\hline Eszopiclone & 1129010 & N05CF04 \\
\hline Zolpidem Tartrate & 1129009 & N05CF02 \\
\hline Zopiclone & 1129007 & N05CF01 \\
\hline \multicolumn{3}{|l|}{ Nervous system: Tricyclic antidepressants } \\
\hline Amitriptyline Hydrochloride & 1179002 & N06AA09 \\
\hline Amoxapine & 1179001 & N06AA17 \\
\hline Clomipramine Hydrochloride & 1174002 & N06AA04 \\
\hline Dosulepin Hydrochloride & 1179027 & N06AA16 \\
\hline Imipramine Hydrochloride & 1174006 & N06AA02 \\
\hline Lofepramine Hydrochloride & 1174004 & N06AA07 \\
\hline Nortriptyline Hydrochloride & 1179004 & N06AA10 \\
\hline Trimipramine Maleate & 1174005 & N06AA06 \\
\hline \multicolumn{3}{|c|}{ Nervous system: Selective serotonin reuptake inhibitor (SSRI) } \\
\hline Escitalopram Oxalate & 1179054 & N06AB10 \\
\hline Fluvoxamine Maleate & 1179039 & N06AB08 \\
\hline Paroxetine Hydrochloride Hydrate & 1179041 & N06AB05 \\
\hline Sertraline Hydrochloride & 1179046 & N06AB06 \\
\hline \multicolumn{3}{|c|}{ Nervous system: Antiparkinsonian drugs - Anticholinergic drugs } \\
\hline Biperiden Hydrochloride & 1162001 & N04AA02 \\
\hline Mazaticol Hydrochloride Hydrate & 1169004 & N04AA10 \\
\hline Piroheptine Hydrochloride & 1169003 & N04AA \\
\hline Profenamine Hibenzate & 1163002 & N04AA05 \\
\hline Profenamine Hydrochloride & 1163001 & N04AA05 \\
\hline
\end{tabular}


Table 3 Proposal for drug coding of "List of drugs to be prescribed with special caution" ${ }^{1)}$ (Continued)

Therapeutic category/JAN English name
Promethazine Hydrochloride
Promethazine Hibenzate
Trihexyphenidyl Hydrochloride
Systemic hormonal preparations, excl. Sex hormones and insulins: Oral corticosteroids

Betamethasone

Cortisone Acetate

Dexamethasone

Hydrocortisone

Methylprednisolone

Prednisolone

Triamcinolone

Combination (see Table 5)

Blood and blood forming organs: Antithrombotic drugs

Acetylsalicylic acid (Aspirin)

Apixaban

Cilostazol

Clopidogrel Sulfate

Dabigatran Etexilate Methanesulfonate

Edoxaban Tosilate Hydrate

Prasugrel Hydrochloride

Rivaroxaban

Ticlopidine Hydrochloride

Ticagrelor

Warfarin potassium

Combination (see Table 5)

Cardiovascular system: Digitalis

Digoxin

2113003

2113004

Metildigoxin

2113005

Cardiovascular system: High-ceiling diuretics

Azosemide

2139008

2139004

2139005

Furosemide

Piretanide

2139007

Torasemide

2139009

2149045

2133001

Spironolactone

$\mathrm{ATC}^{3)}$

R06AD02

D04AA10

R06AD02

D04AA10

R06AD02

D04AA10

N04AA01

H02AB01

H02AB10

D07AB19

H02AB02

H02AB09

H02AB04

H02AB06

H02AB08

N02BA01

B01AC06

B01AF02

B01AC23

B01AC04

B01AE07

B01AF03

B01AC22

B01AF01

B01AC05

B01AC24

B01AA03

C01AA05

C01AA08

C03CA

C03CA02

C03CA01

C03CA03

C03CA04

C03DA04

C03DA01

Cardiovascular system: Beta blocking agents 
Table 3 Proposal for drug coding of "List of drugs to be prescribed with special caution" 1) (Continued)

\begin{tabular}{|c|c|c|}
\hline Therapeutic category/JAN English name & Japan 2) & $\mathrm{ATC}^{3)}$ \\
\hline Alprenolol Hydrochloride & 2123002 & C07AA01 \\
\hline Arotinolol Hydrochloride & 2123014 & C07AA \\
\hline Bufetolol Hydrochloride & 2123006 & C07AA \\
\hline Carteolol Hydrochloride & $\begin{array}{l}2123005 \\
2149025\end{array}$ & $\begin{array}{l}\text { C07AA15 } \\
\text { S01ED05 }\end{array}$ \\
\hline Nadolol & 2123015 & C07AA12 \\
\hline Nipradilol & 2149021 & $\begin{array}{l}\text { C07AA } \\
\text { S01ED }\end{array}$ \\
\hline Pindolol & $\begin{array}{l}2123009 \\
2149011\end{array}$ & C07AA03 \\
\hline Propranolol Hydrochloride & $\begin{array}{l}2123008 \\
2149014\end{array}$ & C07AA05 \\
\hline \multicolumn{3}{|l|}{ Cardiovascular system: Alpha1 blocking agents } \\
\hline Bunazosin Hydrochloride & 2149015 & C02CA \\
\hline Doxazosin Mesilate & 2149026 & C02CA04 \\
\hline Prazosin Hydrochloride & 2149002 & C02CA01 \\
\hline Terazosin Hydrochloride Hydrate & 2149023 & G04CA03 \\
\hline Urapidil & 2149020 & C02CA06 \\
\hline \multicolumn{3}{|c|}{ Respiratory system: $\mathrm{H}_{1}$ receptor antagonists (1st generation) } \\
\hline Alimemazine Tartrate & 4413003 & R06AD01 \\
\hline Chlorpheniramine Maleate & $\begin{array}{l}4419001 \\
4419003\end{array}$ & R06AB04 \\
\hline Clemastine Fumarate & 4419008 & R06AA04 \\
\hline Cyproheptadine Hydrochloride Hydrate & 4419005 & R06AX02 \\
\hline D-chlorpheniramine Maleate & 4419002 & R06AB04 \\
\hline Diphenhidramine & 4411001 & R06AA02 \\
\hline Homochlorcyclizine Hydrochloride & 4419006 & R06AE \\
\hline Hydroxyzine Hydrochloride & 1179005 & N05BB01 \\
\hline Hydroxyzine Pamoate & 1179019 & N05BB01 \\
\hline Promethazine Hibenzate (relisted) & 4413002 & R06AD02 \\
\hline Promethazine Hydrochloride (relisted) & 4413002 & R06AD02 \\
\hline Promethazine Methylenedisalicylate (relisted) & 4413002 & R06AD02 \\
\hline \multicolumn{3}{|l|}{ Combination (see Table 5) } \\
\hline \multicolumn{3}{|c|}{ Alimentary tract and metabolism: $\mathrm{H}_{2}$ receptor antagonists } \\
\hline Cimetidine & 2325001 & A02BA01 \\
\hline Famotidine & 2325003 & A02BA03 \\
\hline Lafutidine & 2325006 & A02BA08 \\
\hline Nizatidine & 2325005 & A02BA04 \\
\hline Ranitidine Hydrochloride & 2325002 & A02BA02 \\
\hline Roxatidine Acetate Hydrochloride & 2325004 & A02BA06 \\
\hline \multicolumn{3}{|c|}{ Alimentary tract and metabolism: Antiemetic agents } \\
\hline Metoclopramide & 2399004 & A03FA01 \\
\hline Promethazine Hibenzate (relisted) & 4413002 & $\begin{array}{l}\text { R06AD02 } \\
\text { D04AA10 }\end{array}$ \\
\hline Promethazine Hydrochloride (relisted) & 4413002 & $\begin{array}{l}\text { R06AD02 } \\
\text { D04AA10 }\end{array}$ \\
\hline Promethazine Methylenedisalicylate (relisted) & 4413002 & R06AD02 \\
\hline
\end{tabular}


Table 3 Proposal for drug coding of "List of drugs to be prescribed with special caution" ${ }^{1)}$ (Continued)

\begin{tabular}{lll}
\hline Therapeutic category/JAN English name & Japan ${ }^{2)}$ & ATC $^{3)}$ \\
\hline & & D04AA10 \\
Alimentary tract and metabolism: Drugs for constipation & & A02AA02 \\
Magnesium Oxide & 2344002 & A06AD02 \\
& 2344009 & A10BA03 \\
Alimentary tract and metabolism: Biguanides & 3962001 & A10BA02 \\
Buformin Hydrochloride & 3962002 & \\
Metformin Hydrochloride & & \\
Combination (see Table 5) & & A10BB31 \\
Alimentary tract and metabolism: sulfonylureas & 3961001 & A10BB02 \\
Acetohexamide & 3961004 & A10BB01 \\
Chlorpropamide & 3961003 & A10BB09 \\
Glibenclamide & 3961007 & A10BB12 \\
Gliclazide & 3961008 & A10BB \\
Glimepiride & 3961002 & A10BB03 \\
Glyclopyramide & 3961006 & \\
Tolbutamide & & \\
\hline
\end{tabular}

Combination (see Table 5)

Alimentary tract and metabolism: Alpha glucosidase inhibitors

Acarbose

Miglitol

Voglibose

3969004

Combination (see Table 5)

Alimentary tract and metabolism: Thiazoridinediones

Pioglitazone Hydrochloride

3969007

A10BG03

Combination (see Table 5)

Alimentary tract and metabolism: Sodium-glucose co-transporter 2 (SGLT2) inhibitors

Canagliflozin Hydrate

3969022

A10BK02

Dapagliflozin Propylene Glycolate Hydrate

3969019

A10BK01

Empagliflozin

3969023

A10BK03

Ipragliflozin L-proline

3969018

A10BK

Luseogliflozin Hydrate

3969020

A10BK

Tofogliflozin Hydrate

3969021

A10BK

Urologicals: Muscarinic receptor antagonists

Fesoterodine Fumarate

2590015

G04BD1 1

Imidafenacin

2590013

G04BD

Oxybutynin Hydrochloride

2590005

G04BD04

Propiverine Hydrochloride

2590007

G04BD06

Solifenacin Succinate

2590011

G04BD08

Tolterodine Tartrate

2590012

G04BD07

Musculo-skeletal system: Non-steroidal antiinflammatory drugs (NSAIDs)

Acemetacin

Amfenac Sodium Hydrate

Ampiroxicam

1149030

M01 AC

Acetylsalicylic acid (Aspirin) (relisted) 
Table 3 Proposal for drug coding of "List of drugs to be prescribed with special caution" 1) (Continued)

\begin{tabular}{|c|c|c|}
\hline Therapeutic category/JAN English name & Japan 2) & $\mathrm{ATC}^{3)}$ \\
\hline Bucolome & 1149009 & M01AX \\
\hline Diclofenac Sodium & 1147002 & $\begin{array}{l}\text { M01AB05 } \\
\text { S01 BC03 } \\
\text { M02AA15 }\end{array}$ \\
\hline Emorfazone & 1148004 & N02BG \\
\hline Etodolac & 1149032 & M01AB08 \\
\hline Flufenamate Aluminum & 1141004 & M01AG03 \\
\hline Flurbiprofen & 1149011 & $\begin{array}{l}\text { M01AE09 } \\
\text { M02AA19 }\end{array}$ \\
\hline Ibuprofen & 1149001 & M01AE01 \\
\hline Indometacin & $\begin{array}{l}1145001 \\
1145002\end{array}$ & $\begin{array}{l}\text { C01EB03 } \\
\text { M01AB01 } \\
\text { M02AA23 }\end{array}$ \\
\hline Indomethacin Farnesil & 1145005 & M01AB01 \\
\hline Lornoxicam & 1149036 & M01 AC05 \\
\hline Loxoprofen Sodium Hydrate & 1149019 & $\begin{array}{l}\text { M01AE } \\
\text { M02AA }\end{array}$ \\
\hline Mefenamic Acid & 1141005 & M01AG01 \\
\hline Meloxicam & 1149035 & M01 ACO6 \\
\hline Mofezolac & 1149033 & M01AX \\
\hline Nabumetone & 1149027 & M01AX01 \\
\hline Naproxen & 1149007 & M01AE02 \\
\hline Oxaprozin & 1149026 & M01AE12 \\
\hline Piroxicam & 1149017 & $\begin{array}{l}\text { M01 AC01 } \\
\text { M02AA07 } \\
\text { S01 BC06 }\end{array}$ \\
\hline Pranoprofen & 1149010 & S01 BC09 \\
\hline Proglumetacin Maleate & 1145004 & M01AB14 \\
\hline Sulindac & 1149015 & M01AB02 \\
\hline Tiaprofenic Acid & 1149025 & M01AE11 \\
\hline Tiaramide Hydrochloride & 1148001 & N02BG \\
\hline Zaltoprofen & 1149029 & M01AE \\
\hline Combination (See Table 5) & & \\
\hline
\end{tabular}

Japanese version available at http://www.jpn-geriat-soc.or.jp/tool/xls/list_03.xlsx or http://www.jpn-geriat-soc.or.jp/tool/pdf/list_02.pdf

ATC: Anatomical Therapeutic Chemical Classification System

JAN: Japanese Accepted Names for Pharmaceuticals

Japan: the first 7-digit numbers of the code of the Japanese drug price list

${ }^{1)}$ Drugs that had been previously approved but do not currently being marketed are excluded. The list includes long-term oral use drugs as a general rule, except self-injection

insulin, according to the guidelines (Japan Geriatric Society. Guidelines for Medical Treatment and its Safety in the elderly 2015 (In Japanese). Toyko, Medical View Co., Ltd. 2015)

${ }^{2)} \mathrm{A}$ different base adduct may or may not require different codes in Japan; hydroxyzine (1179005 for hydrochloride, 1179019 for pamoate) or promethazines (4413002)

3)ATC codes for topical use were excluded, e.g. A07EA Corticosteroids acting locally, D04AA Antihistamines for topical use; defined daily dose (DDD) are not available for most of those

${ }^{4)}$ The guidelines distinguish sulpiride and sultopride from other antipsychotic drugs

${ }^{5)}$ The guidelines distinguish acetyhlsalicylic acid (aspirine) from other antithrombotic drugs

${ }^{6)}$ The guidelines distinguish oxybutynin from other muscarinic receptor antagonists

${ }^{7)}$ The guidelines does not have metildigoxin (oral), however it is marketed and added to the Table 3 from molecular based perspectives

been updated since 1990 and, therefore, numerous new drugs are coded as "others." Based on this system, the National Health Insurance Drug Price List $[16,18]$ provides 7 -digit drug codes at substance level, similar to the ATC 5th level, and 12-digit codes at the product level. In contrast with the ATC process, we extracted the code using the first 7-digit numeric code from the 12-digit alphanumeric code to represent the substance level.

The selected drugs and the corresponding codes, proposed first by Nomura and Yonekawa, were compared with those formulated separately by the Japanese system vendor, Data Horizon Corporation (https://www.dhorizon.co.jp). 
Table 4 Proposal for drug coding of "List of drugs to consider starting" 1)

\begin{tabular}{|c|c|c|}
\hline Therapeutic category/JAN English name & Japan $^{2)}$ & ATC $^{2)}$ \\
\hline \multicolumn{3}{|l|}{ Antiparkinson drugs } \\
\hline \multicolumn{3}{|l|}{ Combination (see Table 5) } \\
\hline \multicolumn{3}{|l|}{ Vaccine: Influenza } \\
\hline Influenza HA Vaccine (A/B) & NA & J07BB02 \\
\hline Adsorbed Influenza Virus Vaccine (H5N1) & NA & J07BB02 \\
\hline \multicolumn{3}{|l|}{ Vaccine: Pneumococcal } \\
\hline Pneumococcal Polysaccharide Conjugate Vaccine(adsorbed) & $631140 G$ & J07AL52 \\
\hline Pneumococcus Vaccine & 6311400 & J07AL01 \\
\hline \multicolumn{3}{|l|}{ Cardiovascular system: Angiotensin conversion enzyme (ACE) inhibitor } \\
\hline Alacepril & 2144003 & C09AA \\
\hline Benazepril Hydrochloride & 2144007 & C09AA07 \\
\hline Captopril & 2144001 & C09AA01 \\
\hline Cilazapril Hydrate & 2144005 & C09AA08 \\
\hline Delapril Hydrochloride & 2144004 & C09AA12 \\
\hline Enalapril Maleate & 2144002 & C09AA02 \\
\hline Imidapril Hydrochloride & 2144008 & C09AA16 \\
\hline Lisinopril Hydrate & 2144006 & C09AA03 \\
\hline Perindopril Erbumine & 2144012 & C09AA04 \\
\hline Quinapril Hydrochloride & 2144010 & C09AA06 \\
\hline Temocapril Hydrochloride & 2144009 & C09AA14 \\
\hline Trandolapril & 2144011 & C09AA10 \\
\hline \multicolumn{3}{|l|}{ Cardiovascular system: Angiotensin receptor blocker (ARB) } \\
\hline Azilsartan & 2149048 & C09CA09 \\
\hline Candesartan Cilexetil & 2149040 & C09CA06 \\
\hline Irbesartan & 2149046 & C09CA04 \\
\hline Olmesartan Medoxomil & 2149044 & C09CA08 \\
\hline Telmisartan & 2149042 & C09CA07 \\
\hline Valsartan & 2149041 & C09CA03 \\
\hline Losartan Potassium & 2149039 & C09CA01 \\
\hline \multicolumn{3}{|l|}{ Coombination (See Table 5) } \\
\hline \multicolumn{3}{|l|}{ Cardiovascular system: Lipid modifying agents (Statine) } \\
\hline Atorvastatin Calcium Hydrate & 2189015 & C10AA05 \\
\hline Fluvastatin Sodium & 2189012 & C10AA04 \\
\hline Pitavastatin Calcium Hydrate & 2189016 & C10AA08 \\
\hline Pravastatin Sodium & 2189010 & C10AA03 \\
\hline Rosuvastatin Calcium & 2189017 & C10AA07 \\
\hline Simvastatin & 2189011 & C10AA01 \\
\hline \multicolumn{3}{|l|}{ Coombination (See Table 5) } \\
\hline \multicolumn{3}{|c|}{ Urologicals: Drugs for benign prostatic hypertrophy (selective alpha-1 blockers) } \\
\hline Naftopidil & 2590009 & G04CA \\
\hline Silodosin & 2590010 & G04CA04 \\
\hline Tamsulosin Hydrochloride & 2590008 & G04CA02 \\
\hline \multicolumn{3}{|c|}{ Antineoplastic and immunomodulating agents: Drugs for rheumatoid arthritis } \\
\hline Actarit & 1149031 & M01CX \\
\hline
\end{tabular}


Table 4 Proposal for drug coding of "List of drugs to consider starting" 1) (Continued)

\begin{tabular}{lll}
\hline Therapeutic category/JAN English name & Japan $^{2)}$ & ATC ${ }^{2)}$ \\
\hline Auranofin & 4420001 & M01CB03 \\
Bucillamine & 4420002 & M01CC02 \\
Iguratimod & 3999031 & M01CX \\
Leflunomide & 3999020 & L04AA13 \\
Lobenzarit Sodium & 1149020 & M01CX \\
Methotrexate & 4222001 & L01BA01 \\
Mizoribine & 3999016 & L04AX03 \\
Salazosulfapyridine & 3999002 & L04AX \\
Tofacitinib Citrate & 6219001 & A07EC01 \\
\hline
\end{tabular}

ATC: Anatomical Therapeutic Chemical Classification System

JAN: Japanese Accepted Names for Pharmaceuticals

${ }^{1)}$ Drugs that had been previously approved but do not currently being marketed are excluded. The list includes long-term oral use drugs as a general rule, except self-injection insulin, according to the guidelines (Japan Geriatric Society. Guidelines for Medical Treatment and its Safety in the elderly 2015 (In Japanese). Toyko, Medical View Co., Ltd. 2015.)

${ }^{2)}$ the first 7-digit numbers of the code of the Japanese drug price list.A different base adduct may or may not require different codes in Japan; hydroxyzine (1179005 for hydrochloride, 1179019 for pamoate) or promethazines (4413002).

${ }^{3)}$ ATC codes for topical use were excluded, e.g. A07EA Corticosteroids acting locally, D04AA Antihistamines for topical use; defined daily dose (DDD) are not available for most of those.

Then, the differences were checked and returned to both the authors and the corporation to reach a consensus.

\section{Results}

The drug list is presented in Tables 3, 4, and 5. Of the 236 encoded drug substances, 197 matched the 5th level of the ATC, along with 10 of 26 combinations. No ATC was available at the 5th level for 39 substances and, therefore, they were identified as 4th level substances. If multiple ATCs at the 5th level were available for one substance, the best pharmacological match or the indication-matched ATC was selected and presented with the rest of the possible ATCs. These lists are available as a PDF and spreadsheet at http:// docrd.jp/ftp_up/STOPP-J\%20List.pdf and http://docrd.jp/ ftp_up/STOPP-J\%20List.xlsx, and also on the JGS web page for the STOPP-J in Japanese, http://www.jpn-geriat-soc.or.jp/ tool/pdf/list_02.pdf and http://www.jpn-geriat-soc.or.jp/tool/ xls/list_03.xlsx. Since the JGS's list was prepared as a support tool for daily medical practices, medicines rarely used or withdrawn were excluded from our list. Medicines used for short-term treatments were also excluded. The STOPP-J shows all Insulin products as drugs to be prescribed with special caution, however, if describing more accurately it recommends prescribers consider to stop sliding scale administration. This indicates that insulins can be prescribed and, therefore, they are excluded from coding.

Combination products were separately listed (Table 5) and were divided into three groups, consisting of one where the combination of exact substances was found in the ATC, such as amlodipine besylate and irbesartan (C09DB05), the ATC representing combination such as levodopa and decarboxylase inhibitor (N04BA02), and another where each substance had an individual ATC.
Among the constituent substances in the latter case, the ATCs were presented only for the JGS listed substances. For example, only aspirin was selected from BUFFERIN Combination Tablet $^{\circ}$ with aspirin, aluminum glycinate, and magnesium carbonate. If the combination product consisted of the same substances in different proportions, different codes were assigned by the price list. For example, combinations of atorvastatin calcium hydrate, and amlodipine besylate were coded 2190101, 2190102, 2190103, or 2109104, depending on their compounding ratio. In addition, drugs can be categorized differently in the Japanese pricing list and have several codes; for example, BUFFERIN Combination Tablet $^{\circ}$ is 1143010 as "antipyretics, analgesics, and anti-inflammatory agents" and 3399100 as "other agents relating to blood and body fluids" All codes are listed in the tables.

\section{Discussion}

Identification and application of STOPP-J drug substances The efforts to reduce inappropriate drug use in elderly patients are likely to have a substantial impact on reducing drug-related morbidity. One major required step is a change in the prescription behavior of physicians, which is influenced by their knowledge and alert systems involving pharmacists, computerized reminders [19], and promotional information from pharmaceutical companies [20]. The current JGS guidelines provide concept and review steps for prescribing to the elderly but do not fully detail specific substances. Thus, our computerized database of standard drug substances, reflecting the STOPP-J with a corresponding coding system, will provide an efficient way to improve physician knowledge about medication for the elderly. 
Table 5 Proposal for coding the combination drugs of "List of drugs to be prescribed with special caution" and "List of drugs to consider starting"

\begin{tabular}{|c|c|c|}
\hline Combination drugs (substance name in JAN) & Japan 1) & $\mathrm{ATC}^{2)}$ \\
\hline \multicolumn{3}{|l|}{ "List of drugs to be prescribed with special caution" } \\
\hline $\begin{array}{l}\text { Chlorpromazine Hydrochloride } \\
\text { Phenobarbital * } \\
\text { Promethazine Hydrochloride }\end{array}$ & $\begin{array}{l}1179100 \\
1179101\end{array}$ & $\begin{array}{l}\text { R06AD52 promethazine, combinations or N05AA01 chlorpromazine and } \\
\text { R06AD02 promethazine }\end{array}$ \\
\hline $\begin{array}{l}\text { tia } \\
\text { Aluminum Glycinate * } \\
\text { Magnesium Carbonate * }\end{array}$ & $\begin{array}{l}1143010 \\
3399100\end{array}$ & N02BA51 acetylsalicylic acid, combinations excl. Psycholeptics \\
\hline $\begin{array}{l}\text { Acetylsalicylic acid (aspirin) } \\
\text { Clopidogrel Sulfate }\end{array}$ & 3399101 & $\begin{array}{l}\text { N02BA51 acetylsalicylic acid, combinations excl. Psycholeptics or B01AC06 } \\
\text { acetylsalicylic acid and B01AC04 clopidogrel }\end{array}$ \\
\hline $\begin{array}{l}\text { Acetylsalicylic acid (aspirin) } \\
\text { Lansoprazole * }\end{array}$ & 3399102 & B01AC56 acetylsalicylic acid, combinations with proton pump inhibitors \\
\hline $\begin{array}{l}\text { Metformin Hydrochloride } \\
\text { Pioglitazone Hydrochloride }\end{array}$ & 3969100 & A10BD05 metformin and pioglitazone \\
\hline $\begin{array}{l}\text { Glimepiride } \\
\text { Pioglitazone Hydrochloride }\end{array}$ & 3969101 & A10BD06 glimepiride and pioglitazone \\
\hline $\begin{array}{l}\text { Mitiglinide } \\
\text { Voglibose }\end{array}$ & 3969102 & $\begin{array}{l}\text { A10BD Combinations of oral blood glucose lowering drugs or A10BX08 } \\
\text { mitiglinide and A10BF03 voglibose }\end{array}$ \\
\hline $\begin{array}{l}\text { Pioglitazone } \\
\text { Alogliptin** }\end{array}$ & 3969103 & A10BD09 pioglitazone and alogliptin \\
\hline $\begin{array}{l}\text { Metformin } \\
\text { Vildagliptin* }\end{array}$ & 3969104 & A10BD08 metformin and vildagliptin \\
\hline $\begin{array}{l}\text { Alogliptin* } \\
\text { Metformin }\end{array}$ & 3969105 & A10BD13 metformin and alogliptin \\
\hline \multicolumn{3}{|l|}{ "List of drugs to consider starting" } \\
\hline $\begin{array}{l}\text { Entacapone * } \\
\text { Carbidopa Hydrate } \\
\text { Levodopa }\end{array}$ & 1169102 & N04BA03 levodopa, decarboxylase inhibitor and COMT inhibitor \\
\hline $\begin{array}{l}\text { Carbidopa Hydrate * } \\
\text { Levodopa }\end{array}$ & 1169101 & N04BA02 levodopa and decarboxylase inhibitor \\
\hline $\begin{array}{l}\text { Benserazide Hydrochloride * } \\
\text { Levodopa }\end{array}$ & 1169100 & N04BA02 levodopa and decarboxylase inhibitor \\
\hline $\begin{array}{l}\text { Azilsartan } \\
\text { Amlodipine Besilate }\end{array}$ & 2149121 & C09CA09 azilsartan medoxomil and C08CA01 amlodipine \\
\hline $\begin{array}{l}\text { Azelnidipine } \\
\text { Olmesartan Medoxomil }\end{array}$ & 2149115 & $\begin{array}{l}\text { C08C selective calcium channel blockers with mainly vascular effects and } \\
\text { C09CA08 olmesartan medoxomil }\end{array}$ \\
\hline $\begin{array}{l}\text { Amlodipine Besilate } \\
\text { Irbesartan }\end{array}$ & 2149118 & C09DB05 irbesartan and amlodipine \\
\hline $\begin{array}{l}\text { Amlodipine Besilate } \\
\text { Candesartan Cilexetil }\end{array}$ & 2149116 & C09DB07 candesartan and amlodipine \\
\hline $\begin{array}{l}\text { Amlodipine Besilate } \\
\text { Telmisartan }\end{array}$ & 2149117 & C09DB04 telmisartan and amlodipine \\
\hline $\begin{array}{l}\text { Amlodipine Besilate } \\
\text { Valsartan }\end{array}$ & 2149114 & C09DB01 valsartan and amlodipine \\
\hline $\begin{array}{l}\text { Irbesartan } \\
\text { Trichlormethiazide }\end{array}$ & 2149119 & C09DA04 irbesartan and diuretics \\
\hline $\begin{array}{l}\text { Candesartan Cilexetil } \\
\text { Hydrochlorothiazide }\end{array}$ & 2149111 & C09DA06 candesartan and diuretics \\
\hline $\begin{array}{l}\text { Cilnidipine } \\
\text { Valsartan }\end{array}$ & 2149120 & C08CA14 cilnidipine and C09CA03 valsartan \\
\hline $\begin{array}{l}\text { Termisartan } \\
\text { Hydrochlorothiazide }\end{array}$ & 2149113 & C09DA07 telmisartan and diuretics \\
\hline Valsartan & 2149112 & C09DA03 valsartan and diuretics \\
\hline
\end{tabular}


Table 5 Proposal for coding the combination drugs of "List of drugs to be prescribed with special caution" and "List of drugs to consider starting" (Continued)

\begin{tabular}{lll}
\hline Combination drugs (substance name in JAN) & Japan $^{1)}$ & ATC $^{2)}$ \\
\hline $\begin{array}{l}\text { Hydrochlorothiazide } \\
\text { Losartan Potassium }\end{array}$ & 2149110 & C09DA01 losartan and diuretics \\
Atorvastatin Calcium Hydrate & 2190101 & C10BX03 atorvastatin and amlodipine \\
Amlodipine Besilate & 219010221901032109104 & \\
\hline
\end{tabular}

ATC: Anatomical Therapeutic Chemical Classification System

JAN: Japanese Accepted Names for Pharmaceuticals

${ }^{11}$ The first 7-digit numbers of the code of the Japanese drug price list. The drugs with a different compounding ratio of active substances need different codes in the National Health Insurance Drug Price Standard in Japan. For example, there are bland medicinal products with acetylsalicylic acid $330 \mathrm{mg}$ for anti-

inflammatory use (1143010) and $81 \mathrm{mg}$ for antiplatelet (3399100)

${ }^{2)}$ Some combination drugs have individual ATC codes

* Substances are excepted from the STOPP-J list (Table 3and 4)

This study revealed that some substances approved in Japan were omitted from the ATC classification system, which was also reported by Groot et al. [8] in reference to the STOPP/START. This may occur when a drug is marketed in Japan only, and the substance or combination is not registered with the WHO Collaborating Center for Drug Statistics. When other countries have the same situation, it would also be necessary to set up the framework to ask the WHO Collaboration Center to include medicinal substances limited to them. This would enhance ATC completeness. To support ATC users, the Uppsala Monitoring Center/WHO Collaborating Centre for International Drug Monitoring does provide the WHO Drug Global with drug information, including Japanese approved drugs and referencing ATC codes at the 5th level, for global pharmacovigilance [21]. Their service supports linking Japanese substances with the ATC, and major global companies use this service for internal databases. It is important to make ATC codes useful in pharmacovigilance and pharmacoepidemiology studies for all Japanese and worldwide drugs and create an official framework to register new substance as soon as possible. This would facilitate drug safety monitoring by pharmaceutical companies and the review of drugs at the class and substance levels. We excluded some medicinal products from the first listing step of the drug indication categories. This paradoxically suggests that researchers run the risk of including appropriately prescribed drugs when extracting data from the drug classification systems.

When the Beers criteria were applied to studies on Japanese elderly patients, hospitalization risk was higher in potentially inappropriate medication users [12] and, in contrast, no association was observed between potentially inappropriate medication use and adverse outcomes [13]. The study using STOPP and START addressed the notion that potentially inappropriate prescribing increased healthcare utilization [22]. Although some drug utilization studies have been reported on the STOPP-J, the future applications of our results to pharmacoepidemiologic clinical studies are worth considering in Japan, similar to a previous study using the Beers criteria in Japan $[12,13]$. The use of large databases has become more sophisticated, and 13 Japanese healthcare databases are acknowledged by other entities [23] (e.g., JMDC Claims Database ${ }^{\bullet}$ [24], which provides the names and ATC codes of drugs prescribed from 2005). Some unlisted domestic databases also exist, including the National Database of Health Insurance Claims and Specific Health Checkups of Japan (NDB), which maintain data records from April 2013 provided by the ministry [25]. Currently, no ATC codes are available in the NDB, but the National Health Insurance Drug Price List codes are provided and, therefore, our proposed codes can be used. Another database is the Japanese Adverse Drug Event Report database (JADER), which records spontaneous reports of adverse events to the regulatory agency and lists drug names in plain text, without codes [26]. MID-NET is another prospective database, which was launched in April 2018 by the regulatory agency [27]. It is noteworthy that global comparisons based on the guidelines for medication in the elderly would be complicated or difficult to analyze because substances and their corresponding codes vary.

Currently, there are many therapeutic guidelines and principles for the proper use of medicines, and different definitions are presented worldwide or even in certain countries. Since those guidelines are to be updated periodically in several years, the guidelines propose their philosophies and examples, without identification of drugs. Therefore, interpretation and practice tend to vary by users. When adopting the guidelines, it is important to first define drugs of interest at the component molecule level; however, papers that do not identify the studied drug names might exist. In this research, with reference to the research method of Groot et al. [8] of Ireland, we presented concrete pharmaceutical molecules intended by the STOPP-J proposed by the JGS and proposed corresponding drug codes to be widely used in Japan. The results of this research are expected to be 
helpful in designing research and validating the actual condition of medical service at a clinical institute. Another important application is to import the drug code list into electronic prescription systems and health information systems so that the system can aid physicians in prescribing cautiously. This application is expected to be used in practice in the near future.

\section{Limitations of using the list}

This study was limited to Japanese drugs for internal use, except insulin, because the JGS guidelines focus on the long-term use of drugs to promote appropriate medications and avoid systemic adverse events in the elderly. The study also excluded drugs mainly used for short-term treatments of less than 1 month, e.g., antipyretics. In addition, based on the JGS, the target population in our list comprised patients older than 75 years who are with or without frailty, which is quite different from other guidelines. The drug list would be useful in research to understand the status of drug prescribing or hypothesize about the trends in total drug use and polypharmacy. However, more information such as dosage regimens and comorbidities is normally required to answer clinical questions. Users also need to consider how to interpret the output. For example, the alerted drug should be able to be monitored or stopped for individual patients. Because the JGS tool is not meant to be a prescription rule, but rather provides information to support physicians' judgment when prescribing, the dosage regimen and underlying diseases should be mentioned. Lastly, a periodic update of the list is critical for efficient use in practice.

This was the first challenge to identify the STOPP-J substances to be coded. Some difficulties were found through the work in the interpretation of the STOPP-J, for example, insulins, and healthcare data users may misunderstand what the guidelines really proposed. In addition, new medicines need to be timely evaluated to determine whether they should be prescribed with special caution or considered for medication.

\section{Conclusion}

The STOPP-J drug list is proposed in this study as a starting point for discussion for researchers. Our consolidated lists can be used for pharmacoepidemiological database studies. Some WHO ATC codes were omitted owing to regionalized drug availability or combination drugs, which must be considered when using or interpreting the present data.

\section{Acknowledgements}

The authors would like to thank JAPIC for their support in extracting drug names, Data Horizon Corporation and Shigeki Zeniya for coding assistance, and Yasuyuki Matsushita and Yoko Yoshimoto for sharing their knowledge on drug coding by Japanese pharmaceutical companies.

\section{Availability of data and materials}

All the data generated or analyzed during this study are included in this published article (and its supplementary information files).

\section{Authors' contributions}

$\mathrm{AKI}, \mathrm{K}$ and I are members of the JGS and selected substances from prescribers' view point. AKA and $\mathrm{N}$ selected substances from the viewpoint of pharmaceuticals data management and supervised $Y$ who searched the codes corresponding to substances. N was a major contributor to writing the manuscript. All authors read and approved the final manuscript.

\section{Consent for publication}

Not applicable.

\section{Competing interests}

The authors declare that they have no competing interests.

\section{Publisher's Note}

Springer Nature remains neutral with regard to jurisdictional claims in published maps and institutional affiliations.

\section{Author details}

'Division of Molecular Epidemiology, Jikei University School of Medicine, Tokyo, Japan. ${ }^{2}$ Department of Geriatric Medicine, the University of Tokyo, Tokyo, Japan. ${ }^{3}$ Public Health and Epidemiology, Meiji Pharmaceutical University, Tokyo, Japan.

Received: 19 September 2017 Accepted: 10 June 2018

Published online: 03 July 2018

\section{References}

1. Cabinet Office. Situation on aging. In: Annual report on the aging society 2016 (in Japanese); 2016. http://www8.cao.go.jp/kourei/whitepaper/w-2016/ zenbun/pdf/1s1s_1.pdf. Accessed 31 Dec 2016.

2. Ministry of Health, Labour and Welfare. Appropriate drug use for the elderly (In Japanese). 2018. http://www.mhlw.go.jp/stf/shingi/other-iyaku.html?tid= 431862. Accessed 29 April 2018.

3. Japan Geriatric Society. Guidelines for Medical Treatment and its Safety in the elderly 2005 (In Japanese). Tokyo: Medical View Co., Ltd; 2005.

4. American Geriatrics Society. 2015 Beers Criteria Update Expert Panel. American Geriatrics Society 2015 updated beers criteria for potentially inappropriate medication use in older adults. J Am Geriatr Soc. 2015;63:2227-46.

5. Japan Geriatric Society. Guidelines for Medical Treatment and its Safety in the elderly 2015 (In Japanese). Tokyo: Medical View Co., Ltd; 2015.

6. Gallagher P, O'Mahony D. STOPP (screening tool of older Persons' potentially inappropriate prescriptions): application to acutely ill elderly patients and comparison with Beers' criteria. Age Ageing. 2008;37:673-9.

7. O'Mahony D, O'Sullivan D, Byrne S, O'Connor M, Ryan C, Gallagher PSTOPP. START criteria for potentially inappropriate prescribing in older people: version 2. Age Ageing. 2015:44:213-8.

8. Groot D, Vries M, Joling K, Campen J, Hugtenburg J, Marum R, et al. Specifying ICD9, ICPC and ATC codes for the STOPP/START criteria: a multidisciplinary consensus panel. Age Ageing. 2014;43:773-8.

9. Pahor M, Chrischilles E, Guralnik J. Drug data coding and analysis in epidemiologic studies. Eur J Epidemiol. 1994;10:405-11.

10. World Health Organization. Classification of diseases (ICD). 2016. http:// www.who.int/classifications/icd/en/. Accessed 30 Dec 2016.

11. The International Council for Harmonisation of Technical Requirements for Pharmaceuticals for Human Use (ICH). MedDRA. http://www.ich.org/ products/meddra.html. Accessed 30 Dec 2016.

12. Akazawa M, Imai H, Igarashi A, Tsutani K. Potentially inappropriate medication use in elderly Japanese patients. Am J Geriatr Pharmacother 2010:8:146-60

13. Ishii S, Kojima T, Ezawa K, Higashi K, Ikebata Y, Takehisa Y, et al. The association of change in medication regimen and use of inappropriate medication based on beers criteria with adverse outcomes in Japanese long-term care facilities. Geriatr Gerontol Int. 2016; https://doi.org/10.1111/ ggi.12761.

14. WHO Collaborating Centre for Drug Statistics Methodology. Structure and principles (Last updated: 2018-02-15). 2018. https://www.whocc.no/atc/ structure_and_principles/. Accessed 14 June 2018. 
15. Kojima T, Mizukami K, Tomita N, Arai H, Ohrui T, Eto M, et al. Screening tool for older persons' appropriate prescriptions for Japanese: report of the Japan geriatrics society working group on "guidelines for medical treatment and its safety in the elderly.". Geriatr Gerontol Int. 2016;16:983-1001. Ministry of Health, Labour and Welfare. National Health Insurance Drug Price List.: oral (in Japanese). February 2017. Available from http://www.mhlw.go.jp/ topics/2017/02/x|s/tp20170215-01_1.xls. Accessed 15 February 2017

16. The European Pharmaceutical Market Research Association (EPhMRA). Anatomical Classification. http://www.ephmra.org/Anatomical-Classification. Accessed 20 March 2017.

17. Ministry of Health, Labour and Welfare. National Health Insurance Drug Price List:: injection (in Japanese). February 2017. Available from http://www.mhlw. go.jp/topics/2017/02/xls/tp20170215-01_2xls. Accessed 15 February 2017.

18. Monane M, Matthias DM, Nagle BA, Kelly MA. Improving prescribing patterns for the elderly through an online drug utilization review intervention: a system linking the physician, pharmacist, and computer. JAMA. 1998;280:1249-52.

19. Kathryn JA, John LS, Amie CB. Patient and physician attitudes and behaviors associated with DTC promotion of prescription drugs: Summary of FDA survey research results. 19 Nov 2004. https://www.fda.gov/downloads/ AboutFDA/CentersOffices/OfficeofMedicalProductsandTobacco/CDER/ UCM600276.pdf. Accessed 21 Mar 2017.

20. Uppsala Monitoring Centre. WHO Drug Portfolio. https://www.who-umc. org/whodrug/whodrug-portfolio/whodrug-global/. Accessed 21 Mar 2017.

21. Moriarty F, Bennett K, Cahir C, et al. Potentially inappropriate prescribing according to STOPP and START and adverse outcomes in communitydwelling older people: a prospective cohort study. Br J Clin Pharmacol. 2016:82:849-57. https://doi.org/10.1111/bcp.12995.

22. DGI, LLC. B.R.ID.G.E. TO DATA interactive map. http://www.bridgetodata. org/Map. Accessed 23 Mar 2017.

23. Japan Medical Data Center. JMDC Claims Database. https://www.jmdc.co.jp/ en/pharma/database.html. Accessed 21 Mar 2017.

24. Ministry of Health, Labour and Welfare. The guidelines for providing National Database of health insurance claims and specific health checkups of Japan (NDB) (in Japanese). 2016. http://www.mhlw.go.jp/file/05-Shingikai12401000-Hokenkyoku-Soumuka/0000135460.pdf. Accessed 04 Feb 2017.

25. Nomura K, Hinomura Y, Kawaguchi G, Matsushita Y, Marui H, Anzai T, et al. Effect of database profile variation on drug safety assessment: an analysis of spontaneous adverse event reports of Japanese cases. Drug Des Dev Ther. 2015;9:3031-41.

26. Takahashi F. Current Situation and Challenges of Medical Information Database Network (MID-NET) Project. Regulatory Science of Medical Products 2015; 5(3).

27. Fick DM, Mion LC, Beers $M H, L$ Waller J. Health outcomes associated with potentially inappropriate medication use in older adults. Res Nurs Health. 2008:31:42-51

\section{Ready to submit your research? Choose BMC and benefit from:}

- fast, convenient online submission

- thorough peer review by experienced researchers in your field

- rapid publication on acceptance

- support for research data, including large and complex data types

- gold Open Access which fosters wider collaboration and increased citations

- maximum visibility for your research: over $100 \mathrm{M}$ website views per year

At BMC, research is always in progress.

Learn more biomedcentral.com/submissions 